\title{
Introduction to the Special Issue on Mobile Service Robotics and Associated Technologies
}

\author{
Manuel Silva \\ School of Engineering of the Polytechnic Institute of Porto \& INESC TEC, \\ Rua Dr. António Bernardino de Almeida, 431 4249-015, Portugal
}

(Received 14 July 2021; Revised 15 July 2021; Accepted 16 July 2021; Published online 20 July 2021)

The popularity of mobile service robotics is steadily growing, and over the last two decades, there has been a significant increase in the application of these machines in distinct fields, indoors, and outdoors. To become truly effective in performing their assigned tasks, these machines involve several associated technologies, namely sensors, actuators, and control systems.

With these considerations in mind, this special issue of the Journal of Artificial Intelligence and Technology presents six stateof-the-art peer-reviewed contributions, ranging from theory to prototyping, and down to the final product development. The six papers included in this special issue cover distinct application topics: $(i)$ legged robotics, including the definition of the optimal leg transfer modes for walking robots and the proposal of walking gait for a planetary exploration legged rover to prevent falls, (ii) inspection robots for different tasks, namely the inspection of wind turbine blades, subsea flexible risers, and reinforced concrete structures, detailing the associated inspection subsystems, and (iii) wearable systems, through the presentation of a sensitivity adjustment technique for an exoskeleton control system.

The guest editor would like to thank the reviewers for their efforts in reviewing the papers, and the authors for their positive responses to the reviewers' comments and suggestions. Also, special thanks are due to the journal editor, Prof. Yinong Chen, and to the members of the editorial board, for their support of this special issue, which is hoped to constitute a valuable addition to scientific and developmental knowledge in mobile service robotics and its associated technologies. Finally, a word of appreciation and acknowledgment is also due to Prof. M. Osman Tokhi, from London South Bank University, for all his collaboration and help in preparing this special issue. 